\title{
Valuation of guaranteed annuity options in affine term structure models
}

\author{
Chi Chiu Chu and Yue Kuen Kwok ${ }^{\star}$ \\ Department of Mathematics, Hong Kong University of Science and Technology \\ Clear Water Bay, Hong Kong
}

\begin{abstract}
We propose three analytic approximation methods for numerical valuation of the guaranteed annuity options in deferred annuity pension policies. The approximation methods include the stochastic duration approach, Edgeworth expansion and analytic approximation in affine diffusions. The payoff structure in the annuity policies is similar to a quanto call option written on a coupon bearing bond. To circumvent the limitations of the onefactor interest rate model, we model the interest rate dynamics by a two-factor affine interest rate term structure model. The numerical accuracy and computational efficiency of these approximation methods are analyzed. We also investigate the value sensitivity of the guaranteed annuity option with respect to different parameters in the pricing model.
\end{abstract}

JEL classification: G13; G23

Keywords: Guaranteed annuity option, affine term structure models, coupon-bond options, stochastic duration, Edgeworth approximation, affine approximation

\section{Introduction}

Under a guaranteed annuity option, an insurer guarantees to covert a policholder's accumulated funds to a life annuity at a fixed rate when the policy matures. If the annuity rates provided under the guarantee are more beneficial to the policyholder than the prevailing rates in the market, then the insurer has to make up the difference. This is one of the many examples of minimum return guarantees (embedded options) in life insurance policies. Pension-type policies with GAO's were popular in UK retirement saving contracts in the 1970's and 1980's. Between 1975 and 1985, UK interest rates were at a high level (typically above 10\%). It was then generally perceived that the GAO's have insignificant value since these options are deeply out-of-the-money. However, for pension-type contracts having a long term, which may last for 30 years or more, the change in financial and other variables may cause the embedded GAO to become an uncontrollable liability. There are a number of factors that contribute to the acute increase in the value of these GAO's. First, the recent UK interest rates become lower so that the annuity value becomes higher.

* Corresponding author. Tel: 852-2358-7418; fax: 852-2358-1643. E-mail address: maykwok@ust.hk 
Second, the accumulated equity value of these contracts may increase substantially with a strong return in the stock market. Third, the improvement of longevity compared to the anticipated mortality assumption. Actually when these contracts were written in the 1970's and 1980's, the GAO reflected an interest rate of around 5\% based on the mortality assumption implicit on the rates. The great improvement in longevity made the GAO more valuable so that the threshold rate at which guarantee kicked in rose from $5 \%$ to $7 \%$ or $8 \%$. Due to the significant increase in liabilities in these pension-type contracts, Equitable Life (a leading UK insurance firm) had to close for new business. Detailed accounts of the issues faced by the issuance of GAO's can be found in the review articles by O'Brien (2001) and Wilkie et al. (2004).

There have been numerous works on the pricing and hedging of GAO's using the option valuation approach. Boyle and Hardy (2003) provide an insightful review on the issues of pricing, reserving and hedging GAO's under interest rate risk, equity risk and mortality risk. Pelsser (2003) shows how to construct a replicating portfolio of interest rate swaptions that replicates the GAO. The swaption is seen to mimick the type of interest rate exposure faced by the GAO issuer. However, his swaption replication technique still faces problems with the equity risk and mortality risk. The nominal amount to be purchased depends on the assumed growth of the policyholder's fund. Biffis and Millossovich (2006) develop a comprehensive valuation framework for pricing GAO. Their model includes stochastic volatility and discontinuous dynamics in the equity value process and stochastic mortality modeling.

The payoff structure of the GAO resembles a quanto call option written on a couponbearing bond. The "quanto" feature appears since the payoff is in units of "stock" (like units of foreign currency) rather than in cash. The moneyness of the option is dependent on the interest rate risk, mortality level and equity performance. Ballotta and Haberman (2003a) apply the one-factor Heath-Jarrow-Morton model to price GAO in unit-linked deferred annuity contracts that are purchased on the grant date by a single premium [with later extension to include stochastic mortality effects (Ballotta and Haberman (2003b))]. However, an one-factor interest rate model would implicitly imply that all forward rates are perfectly correlated. Since pension policies are long term contracts, it is generally known in the literature that a two-factor interest rate model performs much better in hedging long-term interest rate derivatives.

In this paper, we employ a two-factor interest rate model of the affine class (Dai and Singleton, 2000) to characterize the stochastic interest rate dynamics. Similar to BallottaHaberman framework (2003a), we do not incorporate the insurance company expenses, tax effects and pre-retirement death benefits into our model. Also, the mortality risk is assumed to be unsystematic and independent of the equity and interest rate risks. Under the simplicity of the one-factor interest rate model, Ballotta and Haberman (2003a) are able to apply the decomposition technique of Jamshidian (1989) on pricing options on couponbearing bonds, thanks to the observation that the annuity option payoff can be written as 
the payoff generated by a portfolio of zero-coupon bond options with appropriate strike prices. However, since the interest rates become correlated under the two-factor interest rate model, the Jamshidian decomposition technique cannot be applied.

There will be no explict closed form analytic price formula for the GAO when the interest rate dynamics is modeled by a two-factor interest rate model. However, several analytic approximation methods are known in the literature for pricing bond options or swaptions under the multi-factor affine term structure models. One method uses a single zero-coupon bond as a proxy for the original coupon-bearing bond. The approximation error is minimized by choosing the maturity of the zero-coupon bond to be equal to the stochastic duration (Cox et al., 1979; Wei, 1997) of the coupon-bearing bond. Another method makes use of the Edgeworth approximation of the probability distribution of the value of the coupon-bearing bond (Collin-Dufresne and Goldstein, 2002). The third method approximates the conditional distributions of the risk factors in affine diffusions. The exercise probability of the annuity option is approximated through an approximation of the exercise region. This is achieved by the linearization of the exercise region, whose boundary is approximated by a hyperplane. The exercise probability of the annuity option is approximated through an approximation of the exercise region. This is achieved by the linearization of the exercise region, whose boundary is approximated by a hyperplane. One then compute the relevant probabilities needed for pricing options on coupon-bearing bonds by the same numerical method used in the pricing of options on zero-coupon bonds (Singleton and Umantsev, 2002). We adopt and modify these analytic approximation methods for numerical valuation of the GAO in deferred annuity pension policies. The numerical accuracy and computational efficiency of these approximation schemes are compared, and the impact of different parameter values in the pricing model on the GAO value are investigated.

The paper is organized as follows. In the next section, we present the model setup of the GAO and the formulation of the multi-factor affine interest rate model. Nice analytic tractability of the affine term structure model for finding the present value of annuity payments are demonstrated. In Section 3, we discuss the method of minimum variance duration. The GAO is priced under the measure associated with the numeraire that is related to the annuity payment paid at $\tau$-period after retirement. A judicious analytic approximation is made in the expectation calculations so that closed form approximation formula can be obtained. The pricing error between the exact and approximate solutions is minimized by choosing $\tau$ such that the variance of the value of the future stream of annuity payments normalized by the price of the $\tau$-maturity bond is minimized. In Section 4 , we illustrate how to perform the Edgeworth expansion of the distribution of the annuity value at the maturity of the policy. Under the affine diffusion assumption, the bond prices are exponential affine functions of the risk factors. The moments of the annuity value are also exponential affine so that the coefficients can be solved through the solution of a system of Ricatti equations. In Section 5, we apply the affine approximation approach to 
the valuation of GAO. Section 6 reports the numerical experiments that were performed to compare numerical accuracy and computational efficiency of the minimum variance duration approach, Edgeworth series approximation and affine approximation. Pricing behaviors of the GAO are also examined. The last section summarizes and concludes the main results of the paper.

\section{Model setup of the guaranteed annuity option}

The payoff structure of a guaranteed annuity option (GAO) is similar to a call option on a coupon bond, where the "coupons" are the future stream of annuity payments. Besides the interest rate risk as in usual options on a coupon bond, the GAO also has exposure in equity risk and mortality risk. The equity risk arises since the payoff is in units of stock rather than in cash so that the payoff is essentially in the form of a quanto option (the equity risk in GAO resembles the exchange rate risk in quanto option). For the mortality risk, we assume that it is independent of the financial risk so that it is diversifiable. It is quite acceptable to use deterministic mortality for valuing options that are dependent on the death of the policyholder (Boyle and Hardy, 2003).

We consider a single premium equity-linked policy whose policy's maturity date is $T$. The maturity date $T$ coincides with the retirement age $R$ of the policyholder. The premium is invested in equity whose value $S_{t}$ is assumed to follow a Geometric Brownian process. We let $a_{R}(t)$ denote the market value at time $t$ of a life annuity of one dollar per annum starting at age $R$. Let ${ }_{n} p_{R}$ denote the probability that a person aged $R$ survives $n$ years and $D_{T+n}(t)$ denote the market value of the unit par default free zero-coupon bond at time $t$ with maturity date $T+n$. Also, we let $\omega$ denote the maximum age in the mortality table. By constructing a portfolio of default free bonds that match exactly with the expected cash flows of the annuity, the value of annuity $a_{R}(T)$ is given by

$$
a_{R}(T)=\sum_{n=0}^{\omega-R-1}{ }_{n} p_{R} D_{T+n}(T)=1+\sum_{n=1}^{\omega-R-1}{ }_{n} p_{R} D_{T+n}(T),
$$

since ${ }_{0} p_{R}=D_{T}(T)=1$. Provided that the policyholder survives to maturity date $T$, he either receives $S_{T}$ or $\frac{S_{T}}{g} a_{R}(T)$ at $T$, whichever has a high value. Here, $g$ is called the guaranteed conversion rate (say, $g=9$ ). When the policyholder exercises the GAO, the equity fund $S_{T}$ is used to purchase an annuity of $S_{T} / g$. The value of the GAO at maturity $T$ is then given by

$$
\text { terminal value of } \mathrm{GAO}=\frac{S_{T}}{g}\left(a_{R}(T)-g\right)^{+},
$$

where $x^{+}=\max (x, 0)$. By assuming deterministic mortality rates, the payoff of the form $\left(a_{R}(T)-g\right)^{+}$resembles an option on a coupon bond with strike $g$ and coupon payment of amount ${ }_{n} p_{R}$ at time $T+n, n=0,1, \cdots$. The factor $S_{T} / g$ behaves like the exchange rate 
factor in a quanto option. The GAO has two types of financial risk exposure: interest rate risk and equity risk.

When the interest rate dynamics is modeled by an one-factor short rate model, it is relatively straightforward to obtain closed form formula for the GAO using the Jamshidian decomposition technique for coupon bearing bond (Boyle and Hardy, 2003; Ballotta and Haberman, 2003a). Unfortunately, the one-factor assumption of the short rate would imply full correlation of all forward interest rates. Such feature invites criticism when the onefactor short rate model is employed to price long term interest rate derivatives. In this paper, we use the multi-factor affine term structure framework to model the interest rate derivatives. The affine framework has become more popular due to its analytic tractability and flexibility. Also, the multi-factor affine model can be easily calibrated through fitting of the current term structure of traded bond prices. Here, we assume that the evolution of the equity value process follows the usual lognormal distribution, though nowadays it becomes more popular to model equity dynamics using stochastic volatility and discontinuous jump dynamics (Biffis and Millossovich, 2006).

\subsection{Multi-factor affine term structure model}

Let $r_{t}$ denote the short rate. The stochastic processes of $r_{t}$ and the $\ell$-component vector of risk factors $\boldsymbol{x}(t)$ under the risk neutral measure $Q$ (money market account is used as the numeraire) are governed by

$$
\begin{aligned}
r_{t} & =\boldsymbol{a}(t)^{T} \boldsymbol{x}(t)+\boldsymbol{b}(t) \\
d \boldsymbol{x}(t) & =\boldsymbol{\mu}(\boldsymbol{x}, t) d t+\sigma(\boldsymbol{x}, t) d \boldsymbol{Z}(t),
\end{aligned}
$$

where the parameter function

$$
\boldsymbol{a}(t)=\left(\begin{array}{c}
a_{1}(t) \\
a_{2}(t) \\
\vdots \\
a_{\ell}(t)
\end{array}\right)
$$

is a deterministic $\ell$-component vector function, $b(t)$ is a scalar function and

$$
\boldsymbol{\mu}(\boldsymbol{x}, t)=\left(\begin{array}{c}
\mu_{1}(\boldsymbol{x}, t) \\
\mu_{2}(\boldsymbol{x}, t) \\
\vdots \\
\mu_{\ell}(\boldsymbol{x}, t)
\end{array}\right) \quad \text { and } \quad \sigma(\boldsymbol{x}, t)=\left(\begin{array}{ccc}
\sigma_{11}(\boldsymbol{x}, t) & \cdots & \sigma_{1 m}(\boldsymbol{x}, t) \\
\vdots & & \vdots \\
\sigma_{\ell 1}(\boldsymbol{x}, t) & \cdots & \sigma_{\ell m}(\boldsymbol{x}, t)
\end{array}\right)
$$

are the drift rate vector and volatility matrix for $\boldsymbol{x}(t)$. Also, the $m$ components in the random vector

$$
\boldsymbol{Z}(t)=\left(\begin{array}{c}
Z_{1}(t) \\
Z_{2}(t) \\
\vdots \\
Z_{m}(t)
\end{array}\right)
$$


are uncorrelated Wiener processes under the risk neutral measure $Q$. Under certain conditions on $\boldsymbol{\mu}$ and $\sigma$, the time- $t$ value of the zero-coupon bond maturing at time $T$ admits solution that has the following exponential affine form (Dai and Singleton, 2000)

$$
D_{T}(t)=\exp \left(-\boldsymbol{A}_{T}(t)^{T} \boldsymbol{x}(t)-B_{T}(t)\right)
$$

where $\boldsymbol{A}_{T}(t)$ and $B_{T}(t)$ are governed by a system of Ricatti differential equations. To be more precise on the functional dependence, $D_{T}(t)$ is a function of $\boldsymbol{x}, t$ and time to maturity $T-t, \boldsymbol{A}_{T}(t)$ is a function of $T-t$ while $B_{T}(t)$ is a function of both $t$ and $T-t$. The volatility vector of the bond price is given by

$$
\boldsymbol{\sigma}_{D}(\boldsymbol{x}, t ; T)=\left(\begin{array}{c}
\sigma_{D, 1}(\boldsymbol{x}, t ; T) \\
\sigma_{D, 2}(\boldsymbol{x}, t ; T) \\
\vdots \\
\sigma_{D, m}(\boldsymbol{x}, t ; T)
\end{array}\right)=-\sigma(\boldsymbol{x}, t)^{T} \boldsymbol{A}_{T}(t)
$$

For the equity fund, its time- $t$ value under the risk neutral measure $Q$ is modeled by

$$
\frac{d S_{t}}{S_{t}}=\left(r_{t}-q\right) d t+\boldsymbol{\sigma}_{S}(t)^{T} d \boldsymbol{Z}
$$

where $q$ is the constant dividend yield and $\boldsymbol{\sigma}_{S}(t)^{T}=\left(\sigma_{S, 1}(t) \sigma_{S, 2}(t) \cdots \sigma_{S, m}(t)\right)$ is the vector of equity volatilities.

\subsection{Risk neutral valuation of GAO value}

Under the risk neutral measure $Q$, the time- $t$ value $V(S, \boldsymbol{x}, t)$ of the GAO is given by the risk neutral expectation of the payoff at time $T$ times the probability of survival of the policyholder over the next $T-t$ years. The probability of survival is given by ${ }_{T-t} p_{R-(T-t)}$ since the policyholder reaches age $R$ in $T-t$ years later. We assume that the company has well diversified the sale of annuity products so that the mortality risk can be taken to be independent of the financial risk under the risk neutral measure $Q$. Given the terminal payoff defined in Eq. (2.2), the time- $t$ value of the GAO is given by

$$
\begin{aligned}
V(S, \boldsymbol{x}, t)= & { }_{T-t} p_{R-(T-t)} E_{Q}\left[e^{-\int_{t}^{T} r_{u} d u} \frac{S_{T}}{g}\left(a_{R}(T)-g\right)^{+}\right] \\
= & { }_{T-t} p_{R-(T-t)}\left\{E_{Q}\left[e^{-\int_{t}^{T} r_{u} d u} \frac{S_{T}}{g} \sum_{n=1}^{\omega-R-1}{ }_{n} p_{R} D_{T+n}(t) \mathbf{1}_{\left\{a_{R}(T)>g\right\}}\right]\right. \\
& \left.-\left(1-\frac{1}{g}\right) E_{Q}\left[e^{-\int_{t}^{T} r_{u} d u} S_{T} \mathbf{1}_{\left\{a_{R}(T)>g\right\}}\right]\right\} .
\end{aligned}
$$

It will be illustrated that the above expectation calculations can be simplified by the method of change of numeraire. 
Let $F_{n}(S, \boldsymbol{x}, t)$ denote the time- $t$ value of a security that pays $S_{T} D_{T+n}(T)$ at time $T$ so that $F_{n}(S, \boldsymbol{x}, t) / g$ gives the time- $t$ value of the annuity payment at time $T+n$. By using the equity fund value $S_{t}$ as the numeraire, we obtain

$$
\begin{aligned}
F_{n}(S, \boldsymbol{x}, t) & =E_{Q}\left[e^{-\int_{t}^{T} r_{u} d u} S_{T} D_{T+n}(T)\right] \\
& =S_{t} e^{-q(T-t)} E_{Q_{S}}\left[D_{T+n}(T)\right],
\end{aligned}
$$

where $Q_{S}$ is the measure associated with the numeraire $S_{t}$. In a similar manner, the expectation of the second term in Eq. (2.6) can be expressed as

$$
\begin{aligned}
E_{Q}\left[e^{-\int_{t}^{T} r_{u} d u} S_{T} \mathbf{1}_{\left\{a_{R}(T)>g\right\}}\right] & =S_{t} e^{-q(T-t)} E_{Q_{S}}\left[\mathbf{1}_{\left\{a_{R}(T)>g\right\}}\right] \\
& =S_{t} e^{-q(T-t)} P_{Q_{S}}\left[a_{R}(T)>g\right]
\end{aligned}
$$

where $P_{Q_{S}}[A]$ denotes the probability of event $A$ occurring under the measure $Q_{S}$. To compute the expectation of the first term in Eq. (2.6), it is more appropriate to choose $F_{n}(S, \boldsymbol{x}, t), n=1,2, \cdots, \omega-R$, as the numeraire. Let $Q_{F_{n}}$ denote the measure associated with the numeraire $F_{n}$. We then have

$$
\begin{aligned}
& E_{Q}\left[e^{-\int_{t}^{T} r_{u} d u} \frac{S_{T}}{g} \sum_{n=1}^{\omega-R-1}{ }_{n} p_{R} D_{T+n}(T) \mathbf{1}_{\left\{a_{R}(T)>g\right\}}\right] \\
= & \sum_{n=1}^{\omega-R-1} \frac{{ }_{n} p_{R}}{g} F_{n}(S, \boldsymbol{x}, t) P_{Q_{F_{n}}}\left[a_{R}(T)>g\right] .
\end{aligned}
$$

In our subsequent discussion, we limit the multi-factor affine term structure model to the Gaussian type model, where the volatility matrix $\sigma(\boldsymbol{x}, t)$ defined in Eq. (2.3) is a function of $t$ only. For a Gaussian type model, the bond price volatility vector becomes

$$
\boldsymbol{\sigma}_{D}(t ; T)=-\sigma(t)^{T} \boldsymbol{A}_{T}(t)
$$

\subsection{Stochastic differential equations}

Under the risk neutral measure $Q$, the stochastic differential equation (SDE) of $D_{T}(t)$ is given by

$$
\frac{d D_{T}(t)}{D_{T}(t)}=r_{t} d t+\boldsymbol{\sigma}_{D}(t ; T)^{T} d \boldsymbol{Z}
$$

Using the Girsanov Theorem, the SDE of $D_{T}(t)$ under the measure $Q_{S}$ is given by

$$
\frac{d D_{T}(t)}{D_{T}(t)}=\left[r_{t}+\boldsymbol{\sigma}_{D}(t ; T)^{T} \boldsymbol{\sigma}_{S}(t)\right] d t+\boldsymbol{\sigma}_{D}(t ; T)^{T} d \boldsymbol{Z}_{Q_{S}},
$$


where $\boldsymbol{Z}_{Q_{S}}$ is a vector of Brownian processes under $Q_{S}$. The SDE of $F_{n}(S, \boldsymbol{x}, t)$ under the risk neutral measure $Q$ is given by

$$
\begin{aligned}
\frac{d F_{n}}{F_{n}} & =r_{t} d t+\left[\boldsymbol{\sigma}_{S}(t)+\boldsymbol{\sigma}_{D}(t ; T+n)-\boldsymbol{\sigma}_{D}(t ; T)\right]^{T} d \boldsymbol{Z} \\
& =r_{t} d t+\left\{\boldsymbol{\sigma}_{S}(t)+\sigma(t)^{T}\left[\boldsymbol{A}_{T}(t)-\boldsymbol{A}_{T+n}(t)\right]\right\}^{T} d \boldsymbol{Z}
\end{aligned}
$$

Next, we would like to solve for $F_{n}(S, \boldsymbol{x}, t)$. We consider the quantity $\ln \frac{D_{T+n}(t)}{D_{T}(t)}$, whose dynamics under $Q_{S}$ is given by

$$
\begin{aligned}
& d\left(\ln \frac{D_{T+n}(t)}{D_{T}(t)}\right) \\
= & \left\{\boldsymbol{\sigma}_{S}(t)^{T}\left[\boldsymbol{\sigma}_{D}(t ; T+n)-\boldsymbol{\sigma}_{D}(t, T)\right]-\frac{1}{2}\left(\left\|\boldsymbol{\sigma}_{D}(t ; T+n)\right\|^{2}-\left\|\boldsymbol{\sigma}_{D}(t ; T)\right\|^{2}\right)\right\} d t \\
& +\left[\boldsymbol{\sigma}_{D}(t ; T+n)-\boldsymbol{\sigma}_{D}(t ; T)\right]^{T} d \boldsymbol{Z}_{Q_{S}} \\
= & \left\{\left[\boldsymbol{\sigma}_{S}(t)-\boldsymbol{\sigma}_{D}(t ; T)\right]^{T}\left[\boldsymbol{\sigma}_{D}(t ; T+n)-\boldsymbol{\sigma}_{D}(t ; T)\right]-\frac{1}{2}\left\|\boldsymbol{\sigma}_{D}(t ; T+n)-\boldsymbol{\sigma}_{D}(t ; T)\right\|^{2}\right\} d t \\
& +\left[\boldsymbol{\sigma}_{D}(t ; T+n)-\boldsymbol{\sigma}_{D}(t ; T)\right]^{T} d \boldsymbol{Z}_{Q_{S}} .
\end{aligned}
$$

The solution to $F_{n}(S, \boldsymbol{x}, t)$ is readily found to be

$$
\begin{aligned}
F_{n}(S, \boldsymbol{x}, t)= & \frac{D_{T+n}(t)}{D_{T}(t)} S_{t} e^{-q(T-t)} \\
& \exp \left(\int_{t}^{T}\left[\boldsymbol{\sigma}_{S}(u)-\boldsymbol{\sigma}_{D}(u ; T)\right]^{T}\left[\boldsymbol{\sigma}_{D}(u ; T+n)-\boldsymbol{\sigma}_{D}(u ; T)\right] d u\right)
\end{aligned}
$$

Lastly, the SDE of $\boldsymbol{x}$ under $Q_{F_{n}}$ can be deduced to be

$$
\begin{aligned}
d \boldsymbol{x} & =\left\{\boldsymbol{\mu}(\boldsymbol{x}, t)+\sigma(t)\left[\boldsymbol{\sigma}_{S}(t)+\boldsymbol{\sigma}_{D}(t ; T+n)-\boldsymbol{\sigma}_{D}(t ; T)\right]\right\} d t+\sigma(t) d \boldsymbol{Z}_{Q_{F_{n}}} \\
& =\left\{\boldsymbol{\mu}(\boldsymbol{x}, t)+\sigma(t) \boldsymbol{\sigma}_{S}(t)+\sigma(t) \sigma(t)^{T}\left[\boldsymbol{A}_{T}(t)-\boldsymbol{A}_{T+n}(t)\right]\right\} d t+\sigma(t) d \boldsymbol{Z}_{Q_{F_{n}}},
\end{aligned}
$$

where $\boldsymbol{Z}_{Q_{F_{n}}}$ is a vector of Brownian processes under the measure $Q_{F_{n}}$.

Under the Gaussian type affine term structure model, the bond prices are lognormally distributed [see Eq. (2.4a,b)]. Since the future annuity payment stream can be visualized as a portfolio of discount bonds and the density of the sum of lognormal distributions has no closed form representation, so there is no closed form analytic solution to the GAO value under the multi-factor affine term structure model. In the next three sections, we explore three different analytic approximation methods for finding approximate solution to $V(S, \boldsymbol{x}, t)$. 


\section{Method of minimum variance duration}

We adopt the idea of minimum variance duration similar to that proposed by Munk (1999). The minimum variance duration approach has been shown to give highly accurate approximation solution to an option on coupon bearing bond under the multi-factor interest rate model. Recall that the stochastic duration of a coupon bearing bond in a multi-factor diffusion model is defined to be the time to maturity of the zero-coupon bond with the same relative volatility as that of the coupon bearing bond (Wei, 1997). The minimum variance duration may be considered as an extension of the concept of stochastic duration.

The solution of the GAO value may be sought by pricing under the measure associated with the numeraire corresponding to the security that pays $S_{T} a_{R}(T)$ at $T$. However, the pricing under such measure is not analytically tractable. Instead, we consider an alternative numeraire that corresponds to the security that pays $S_{T} D_{T+\tau}(T)$ at maturity time $T$. Here, $\tau$ represents the time to maturity of the underlying bond at time $T$. Later, we illustrate how to choose the parameter $\tau$ such that the error in the approximate solution is minimized in some sense. Let $F_{\tau}(S, \boldsymbol{x}, t)$ denote the time- $t$ value of such security and $Q_{F_{\tau}}$ denote the pricing measure when $F_{\tau}(S, \boldsymbol{x}, t)$ is used as the numeraire. Under $Q_{F_{\tau}}$, the time- $t$ value of the GAO is given by [see Eqs. (2.6) and (2.9)]

$$
\begin{aligned}
V(S, \boldsymbol{x}, t) & ={ }_{T-t} p_{R-(T-t)} E_{Q}\left[e^{-\int_{t}^{T} r_{u} d u} S_{T}\left(\frac{a_{R}(T)}{g}-1\right)^{+}\right] \\
& ={ }_{T-t} p_{R-(T-t)} F_{\tau}(S, \boldsymbol{x}, t) E_{Q_{F_{\tau}}}\left[\left(\frac{a_{R}(T)}{g D_{T+\tau}(T)}-\frac{1}{D_{T+\tau}(T)}\right)^{+}\right] .
\end{aligned}
$$

Nice analytic tractability can be achieved if we set $\frac{a_{R}(T)}{g D_{T+\tau}(T)}$ be some constant $K$. Here, $K$ is judiciously chosen to be the mean of $\frac{a_{R}(T)}{g D_{T+\tau}(T)}$ under $Q_{F_{\tau}}$. The analytic approximate solution to $V(S, \boldsymbol{x}, t)$ is taken to be

$$
V_{a}(S, \boldsymbol{x}, t)={ }_{T-t} p_{R-(T-t)} F_{\tau}(S, \boldsymbol{x}, t) E_{Q_{F_{\tau}}}\left[\left(K-\frac{1}{D_{T+\tau}(T)}\right)^{+}\right],
$$

where

$$
K=E_{Q_{F_{\tau}}}\left[\frac{a_{R}(T)}{g D_{T+\tau}(T)}\right]
$$

The remaining analytic procedures include (i) the derivation of closed form analytic expression for $V_{a}(S, \boldsymbol{x}, t)$, (ii) the determination of the parameter $\tau$ such that the pricing error $\left|V(S, \boldsymbol{x}, t)-V_{a}(S, \boldsymbol{x}, t)\right|$ is minimized based on the minimization of variance.

\subsection{Approximate price formula}


First, the mean $K$ can be readily found to be

$$
\begin{aligned}
K & =\frac{1}{g} \sum_{n=0}^{\omega-R-1}{ }_{n} p_{R} E_{Q_{F_{\tau}}}\left[\frac{D_{T+n}(T)}{D_{T+\tau}(T)}\right] \\
& =\frac{1}{g} \frac{S_{t} e^{-q(T-t)}}{F_{\tau}(S, \boldsymbol{x}, t)} \sum_{n=0}^{\omega-R-1}{ }_{n} p_{R} E_{Q_{S}}\left[D_{T+n}(T)\right] \\
& =\frac{1}{g F_{\tau}(S, \boldsymbol{x}, t)} \sum_{n=0}^{\omega-R-1}{ }_{n} p_{R} F_{n}(S, \boldsymbol{x}, t) .
\end{aligned}
$$

Next, the expectation in Eq. (3.2a) is found to be

$$
\begin{aligned}
& E_{Q_{F_{\tau}}}\left[\left(K-\frac{1}{D_{T+\tau}(T)}\right)^{+}\right] \\
= & K P_{Q_{F_{\tau}}}\left[D_{T+\tau}(T)>\frac{1}{K}\right]-\frac{S_{t} e^{-q(T-t)}}{F_{\tau}(S, \boldsymbol{x}, t)} P_{Q_{S}}\left[D_{T+\tau}(T)>\frac{1}{K}\right] .
\end{aligned}
$$

By combining Eqs. (3.3) and (3.4) together, we obtain

$$
\begin{aligned}
V_{a}(S, \boldsymbol{x}, t)= & { }_{T-t} p_{R-(T-t)}\left\{\sum_{n=0}^{\omega-R-1} \frac{{ }_{n} p_{R} F_{n}(S, \boldsymbol{x}, t)}{g} P_{Q_{F_{\tau}}}\left[D_{T+\tau}(T)>\frac{1}{K}\right]\right. \\
& \left.-S_{t} e^{-q(T-t)} P_{Q_{S}}\left[D_{T+\tau}(T)>\frac{1}{K}\right]\right\} .
\end{aligned}
$$

Similar to Eq. (2.11), the dynamics of $\ln \frac{D_{T+\tau}(t)}{D_{T}(t)}$ under $Q_{F_{\tau}}$ is found to be

$$
\begin{aligned}
d\left(\ln \frac{D_{T+\tau}(t)}{D_{T}(t)}\right)= & \left\{\left[\boldsymbol{\sigma}_{S}(t)-\boldsymbol{\sigma}_{D}(t ; T)\right]^{T}\left[\boldsymbol{\sigma}_{D}(t ; T+\tau)-\boldsymbol{\sigma}_{D}(t ; T)\right]\right. \\
& \left.+\frac{1}{2}\left\|\boldsymbol{\sigma}_{D}(t ; T+\tau)-\boldsymbol{\sigma}_{D}(t ; T)\right\|^{2}\right\} d t \\
& +\left[\boldsymbol{\sigma}_{D}(t ; T+\tau)-\boldsymbol{\sigma}_{D}(t ; T)\right]^{T} d \boldsymbol{Z}_{Q_{F_{\tau}}}
\end{aligned}
$$

The mean of $\ln D_{T+\tau}(T)$ under $Q_{F_{\tau}}$ and $Q_{S}$ are obtained as follows:

$$
\begin{aligned}
& E_{Q_{F_{\tau}}}\left[\ln D_{T+\tau}(T)\right]=\bar{c}(\tau)+\frac{\bar{v}^{2}(\tau)}{2}+\ln \left[\frac{D_{T+\tau}(t)}{D_{T}(t)}\right] \\
& E_{Q_{S}}\left[\ln D_{T+\tau}(T)\right]=\bar{c}(\tau)-\frac{\bar{v}^{2}(\tau)}{2}+\ln \left[\frac{D_{T+\tau}(t)}{D_{T}(t)}\right]
\end{aligned}
$$


where

$$
\begin{aligned}
\bar{c}(\tau) & =\int_{t}^{T}\left[\boldsymbol{\sigma}_{S}(u)-\boldsymbol{\sigma}_{D}(u ; T)\right]^{T}\left[\boldsymbol{\sigma}_{D}(u ; T+\tau)-\boldsymbol{\sigma}_{D}(u ; T)\right] d u \\
\bar{v}^{2}(\tau) & =\operatorname{var}\left[\ln D_{T+\tau}(T)\right]=\int_{t}^{T}\left\|\boldsymbol{\sigma}_{D}(u ; T+\tau)-\boldsymbol{\sigma}_{D}(u ; T)\right\|^{2} d u
\end{aligned}
$$

Also, we may express $F_{n}(S, \boldsymbol{x}, t)$ and $K$ in the following forms:

$$
\begin{aligned}
& F_{n}(S, \boldsymbol{x}, t)=\frac{D_{T+n}(t) S_{t}}{D_{T}(t)} e^{-q(T-t)+\bar{c}(n)} \\
& K=\frac{e^{-\bar{c}(\tau)}}{g D_{T+\tau}(t)} \sum_{n=0}^{\omega-R-1}{ }_{n} p_{R} D_{T+n}(t) e^{\bar{c}(n)}=\frac{e^{-\bar{c}(\tau)}}{g D_{T+\tau}(t)} \bar{a}_{R}(t) .
\end{aligned}
$$

Here, the quantity

$$
\bar{a}_{R}(t)=\sum_{n=0}^{\omega-R-1}{ }_{n} p_{R} D_{T+n}(t) e^{\bar{c}(n)}
$$

can be interpreted as the equity-risk-adjusted annuity. Next, we compute the two probability values in Eq. (3.4) and obtain

$$
P_{Q_{F_{\tau}}}\left[D_{T+\tau}(T)>\frac{1}{K}\right]=N\left(-\frac{\ln \frac{1}{K}-\ln \frac{D_{T+\tau}(t)}{D_{T}(t)}-\bar{c}(\tau)-\frac{\bar{v}^{2}(\tau)}{2}}{\bar{v}(\tau)}\right)=N(d)
$$

and

$$
P_{Q_{S}}\left[D_{T+\tau}(T)>\frac{1}{K}\right]=N(d-\bar{v}(\tau))
$$

where

$$
d=\frac{\ln \frac{\bar{a}_{R}(t)}{g D_{T}(t)}+\frac{\bar{v}^{2}(\tau)}{2}}{\bar{v}(\tau)}
$$

Finally, the analytic expression for $V_{a}(S, \boldsymbol{x}, t)$ is found to be

$$
V_{a}(S, \boldsymbol{x}, t)={ }_{T-t} p_{R-(T-t)} S e^{-q(T-t)}\left[\frac{\bar{a}_{R}(t)}{g D_{T}(t)} N(d)-N(d-\bar{v}(\tau))\right] .
$$

\subsection{Determination of $\tau$ using minimization of variance duration}


The error in the approximation of $V(S, \boldsymbol{x}, t)$ by $V_{a}(S, \boldsymbol{x}, t)$ is quantified by $E_{Q_{F_{\tau}}}[|Y|]$, where

$$
Y=\left(\frac{a_{R}(T)}{g D_{T+\tau}(T)}-\frac{1}{D_{T+\tau}(T)}\right)^{+}-\left(K-\frac{1}{D_{T+\tau}(T)}\right)^{+} .
$$

Following a similar approach as proposed by Munk (2000), the pricing error is minimized by choosing $\tau$ so as to minimize the variance of $\frac{d a_{R}(t)}{a_{R}(t)}-\frac{d D_{T+\tau}(t)}{D_{T+\tau}(t)}$. That is, the optimal value of $\tau$ is given by

$$
\tau^{*}=\underset{\tau \geq 0}{\operatorname{argmin}}\left\|\operatorname{var}_{Q_{F_{\tau}}}\left(\frac{d a_{R}(t)}{a_{R}(t)}-\frac{d D_{T+\tau}(t)}{D_{T+\tau}(t)}\right)\right\| .
$$

First we give the justification of the above argument. Afterwards, we present the analytic procedures to obtain $\tau^{*}$.

Let $\widehat{m}=\min \left(\frac{a_{R}(T)}{g D_{T+\tau}(T)}, K\right)$ and $\widehat{M}=\max \left(\frac{a_{R}(T)}{g D_{T+\tau}(T)}, K\right)$. The following three events are mutually exclusive and exhaustive:

$E_{1}=\left\{\frac{1}{D_{T+\tau}(T)} \geq \widehat{M}\right\}, \quad E_{2}=\left\{\widehat{m}<\frac{1}{D_{T+\tau}(T)}<\widehat{M}\right\} \quad$ and $\quad E_{3}=\left\{\frac{1}{D_{T+\tau}(T)} \leq \widehat{m}\right\}$, and from which we deduce that

$$
E_{Q_{F_{\tau}}}[|Y|]=E_{Q_{F_{\tau}}}\left[|Y| \mathbf{1}_{E_{1}}\right]+E_{Q_{F_{\tau}}}\left[|Y| \mathbf{1}_{E_{2}}\right]+E_{Q_{F_{\tau}}}\left[|Y| \mathbf{1}_{E_{3}}\right] \text {. }
$$

Each of the above expectation calculations is analyzed below.

(i) $E_{Q_{F_{\tau}}}\left[|Y| \mathbf{1}_{E_{1}}\right]=0$ since $Y$ becomes zero when $E_{1}$ occurs.

(ii) $E_{Q_{F_{\tau}}}\left[|Y| \mathbf{1}_{E_{2}}\right] \leq\left\{E_{Q_{F_{\tau}}}\left[|Y|^{2}\right] P_{Q_{F_{\tau}}}\left[E_{2}\right]\right\}^{1 / 2}$ and $P_{Q_{F_{\tau}}}\left[E_{2}\right]$ has a smaller value when $\frac{a_{R}(T)}{g D_{T+\tau}(T)}$ stays closer to its mean $K$. This occurs when $\operatorname{var}_{Q_{F_{\tau}}}\left(\frac{a_{R}(T)}{g D_{T+\tau}(T)}\right)$ is minimized.

(iii)

$$
\begin{aligned}
E_{Q_{F_{\tau}}}\left[|Y| \mathbf{1}_{E_{3}}\right] & =E_{Q_{F_{\tau}}}\left[\left|\frac{a_{R}(T)}{g D_{T+\tau}(T)}-K\right| \mathbf{1}_{E_{3}}\right] \\
& \leq\left\{E_{Q_{F_{\tau}}}\left[\left(\frac{a_{R}(T)}{g D_{T+\tau}(T)}-K\right)^{2}\right] P_{Q_{F_{\tau}}}\left[E_{3}\right]\right\}^{1 / 2} \\
& =\left\{\operatorname{var}_{Q_{F_{\tau}}}\left(\frac{a_{R}(T)}{g D_{T+\tau}(T)}\right) P_{Q_{F_{\tau}}}\left[E_{3}\right]\right\}^{1 / 2} .
\end{aligned}
$$

Therefore, one can minimize the pricing error by minimizing $\operatorname{var}{Q_{F_{\tau}}}\left(\frac{a_{R}(T)}{g D_{T+\tau}(T)}\right)$ over the choice of $\tau$. However, the minimization procedure appears to be intractable due 
to the complex expressions for $a_{R}(T)$ and $D_{T+\tau}(T)$. Instead, we attempt to minimize the relative change of value in $\frac{a_{R}(T)}{D_{T+\tau}(T)}$, which can be measured by the variance of $\frac{d a_{R}(t)}{a_{R}(t)}-\frac{d D_{T+\tau}(t)}{D_{T+\tau}(t)}$

Under the risk neutral measure $Q$, the dynamics of $a_{R}(t)$ and $D_{T+\tau}(t)$ are given by

$$
\begin{aligned}
& \frac{d a_{R}(t)}{a_{R}(t)}=r_{t} d t+\boldsymbol{\sigma}_{a}(t ; T)^{T} d \boldsymbol{Z} \\
& \frac{d D_{T+\tau}(t)}{D_{T+\tau}(t)}=r_{t} d t+\boldsymbol{\sigma}_{D}(t ; T+\tau)^{T} d \boldsymbol{Z}
\end{aligned}
$$

where the volatility vector of annuity $\boldsymbol{\sigma}_{a}$ is given by

$$
\boldsymbol{\sigma}_{a}(t ; T)=\sum_{n=0}^{\omega-R-1} \frac{{ }_{n} p_{R} D_{T+n}(t)}{a_{R}(t)} \boldsymbol{\sigma}_{D}(t ; T+n) .
$$

For an one-factor interest rate model, it is readily seen that the solution to $\tau^{*}$ defined in Eq. (3.10) is given by

$$
\boldsymbol{\sigma}_{a}(t ; T)=\boldsymbol{\sigma}_{D}\left(t ; T+\tau^{*}\right)
$$

which is just the stochastic duration of the annuity (Wei, 1997). For the general multifactor case, the minimization of $\operatorname{var}_{Q_{F_{\tau}}}\left(\frac{d a_{R}(t)}{a_{R}(t)}-\frac{d D_{T+\tau}(t)}{D_{T+\tau}(t)}\right)$ leads to the following nonlinear algebraic equation for $\tau$ :

$$
\left[\boldsymbol{\sigma}_{a}(t ; T)-\boldsymbol{\sigma}_{D}(t ; T+\tau)\right]^{T} \frac{\partial \boldsymbol{\sigma}_{D}(t ; T+\tau)}{\partial \tau}=0
$$

\subsection{Two-factor Gaussian model}

We illustrate how to compute $V_{a}(S, \boldsymbol{x}, t)$ using the two-factor Gaussian interest rate model $(G 2++)$ as an example. For the $G 2++$ model, the interest rate $r_{t}$ is given by

$$
r_{t}=x_{1, t}+x_{2, t}+b(t)
$$

where the dynamics of the risk factors are governed by

$$
\begin{aligned}
& d x_{1}=-\kappa_{1} x_{1} d t+\sigma_{1} d Z_{1} \\
& d x_{2}=-\kappa_{2} x_{2} d t+\sigma_{2}\left(\rho d Z_{1}+\sqrt{1-\rho^{2}} d Z_{2}\right) .
\end{aligned}
$$


Here, $b(t)$ is a function which is determined by fitting the current interest rate term structure and $\rho$ is the correlation coefficient between the risk factors. For the $G 2++$ model, the corresponding solution of $\boldsymbol{A}_{T}(t)$ and $B_{T}(t)$ as defined in Eq. (2.4a) are found to be

$$
\boldsymbol{A}_{T}(t)=\left(\begin{array}{c}
\frac{1-e^{-\kappa_{1}(T-t)}}{\kappa_{1}} \\
\frac{1-e^{-\kappa_{2}(T-t)}}{\kappa_{2}}
\end{array}\right)
$$

and

$$
\begin{aligned}
B_{T}(t)= & -\ln \frac{D_{T}(0)}{D_{t}(0)}-\frac{\sigma_{1}^{2}}{\kappa_{1}}\left(\frac{1-e^{-2 \kappa_{1} t}}{2 \kappa_{1}}\right)\left[\frac{1-e^{-2 \kappa_{1}(T-t)}}{2 \kappa_{1}}\right] \\
& -\frac{\sigma_{2}^{2}}{\kappa_{2}}\left(\frac{1-e^{-2 \kappa_{2} t}}{2 \kappa_{2}}\right)\left[\frac{1-e^{-2 \kappa_{2}(T-t)}}{2 \kappa_{2}}\right] \\
& -\rho \sigma_{1} \sigma_{2}\left(\frac{1}{\kappa_{1}}+\frac{1}{\kappa_{2}}\right)\left(\frac{1-e^{-\left(\kappa_{1}+\kappa_{2}\right) t}}{\kappa_{1}+\kappa_{2}}\right)\left[\frac{1-e^{-\left(\kappa_{1}+\kappa_{2}\right)(T-t)}}{\kappa_{1}+\kappa_{2}}\right] \\
& +\left(\frac{\sigma_{1}^{2}}{\kappa_{1}}+\frac{\rho \sigma_{1} \sigma_{2}}{\kappa_{2}}\right)\left(\frac{1-e^{-\kappa_{1} t}}{\kappa_{1}}\right)\left[\frac{1-e^{-\kappa_{1}(T-t)}}{\kappa_{1}}\right] \\
& +\left(\frac{\rho \sigma_{1} \sigma_{2}}{\kappa_{1}}+\frac{\sigma_{2}^{2}}{\kappa_{2}}\right)\left(\frac{1-e^{-\kappa_{2} t}}{\kappa_{2}}\right)\left[\frac{1-e^{-\kappa_{2}(T-t)}}{\kappa_{2}}\right] .
\end{aligned}
$$

Once $\boldsymbol{A}_{T}(t)$ and $B_{T}(t)$ are known, the bond prices $D_{T+n}(t)$ and $\bar{a}_{R}(t)$ can be determined. It remains to find $\bar{c}(\tau)$ and $\bar{v}^{2}(\tau)$ for the G2++ model. The corresponding volatility vector $\boldsymbol{\sigma}_{D}(t ; T)$ is given by

$$
\boldsymbol{\sigma}_{D}(t ; T)=-\left(\begin{array}{c}
\frac{\sigma_{1}}{\kappa_{1}}\left[1-e^{-\kappa_{1}(T-t)}\right]+\frac{\rho \sigma_{2}}{\kappa_{2}}\left[1-e^{-\kappa_{2}(T-t)}\right] \\
\frac{\sigma_{2} \sqrt{1-\rho^{2}}}{\kappa_{2}}\left[1-e^{-\kappa_{2}(T-t)}\right] \\
0
\end{array}\right)
$$

Suppose the stochastic component of the equity fund value $S_{t}$ under the risk neutral measure is $\sigma_{S} d Z_{S}$, where $d Z_{S} d Z_{1}=\rho_{S 1} d t$ and $d Z_{S} d Z_{2}=\rho_{S 2} d t$, then

$$
\frac{d S_{t}}{S_{t}}=(r-q) d t+\boldsymbol{\sigma}_{S}^{T} d \boldsymbol{Z}
$$

where the volatility vector $\boldsymbol{\sigma}_{S}$ is given by

$$
\boldsymbol{\sigma}_{S}^{T}=\left(\begin{array}{lll}
\sigma_{S} \rho_{S 1} & \sigma_{S} \rho_{S 2} & \sigma_{S} \sqrt{1-\rho_{S 1}^{2}-\rho_{S 2}^{2}}
\end{array}\right) .
$$

For the $G 2++$ model, we obtain

$$
\bar{c}(\tau)=\int_{t}^{T}\left[\boldsymbol{\sigma}_{S}-\boldsymbol{\sigma}_{D}(u ; T)\right]^{T}\left[\boldsymbol{\sigma}_{D}(u ; T+\tau)-\boldsymbol{\sigma}_{D}(u ; T)\right] d u
$$




$$
\begin{aligned}
= & \frac{\sigma_{1}^{2}}{\kappa_{1}}\left(\frac{1-e^{-\kappa_{1} \tau}}{\kappa_{1}}\right)\left[\frac{1-e^{-2 \kappa_{1}(T-t)}}{2 \kappa_{1}}\right]+\frac{\sigma_{2}^{2}}{\kappa_{2}}\left(\frac{1-e^{-\kappa_{2} \tau}}{\kappa_{2}}\right)\left[\frac{1-e^{-2 \kappa_{2}(T-t)}}{2 \kappa_{2}}\right] \\
& +\rho \sigma_{1} \sigma_{2}\left(\frac{2-e^{-\kappa_{1} \tau}-e^{-\kappa_{2} \tau}}{\kappa_{1} \kappa_{2}}\right)\left[\frac{1-e^{-\left(\kappa_{1}+\kappa_{2}\right)(T-t)}}{\kappa_{1}+\kappa_{2}}\right] \\
& -\left(\frac{\sigma_{1}^{2}}{\kappa_{1}}+\frac{\rho \sigma_{1} \sigma_{2}}{\kappa_{2}}+\sigma_{1} \sigma_{S} \rho_{S 1}\right)\left(\frac{1-e^{-\kappa_{1} \tau}}{\kappa_{1}}\right)\left[\frac{1-e^{-\kappa_{1}(T-t)}}{\kappa_{1}}\right] \\
& -\left[\frac{\sigma_{2}^{2}}{\kappa_{2}}+\frac{\rho \sigma_{1} \sigma_{2}}{\kappa_{1}}+\sigma_{2} \sigma_{S}\left(\rho_{S 1} \rho+\rho_{S 2} \sqrt{1-\rho^{2}}\right)\right]\left(\frac{1-e^{-\kappa_{2} \tau}}{\kappa_{2}}\right)\left[\frac{1-e^{-\kappa_{2}(T-t)}}{\kappa_{2}}\right],
\end{aligned}
$$

and

$$
\begin{aligned}
& \bar{v}^{2}(\tau)=\int_{t}^{T}\left\|\boldsymbol{\sigma}_{D}(u, T+\tau)-\boldsymbol{\sigma}_{D}(u, T)\right\|^{2} d u \\
= & \sigma_{1}^{2}\left(\frac{1-e^{-\kappa_{1} \tau}}{\kappa_{1}}\right)^{2}\left[\frac{1-e^{-2 \kappa_{1}(T-t)}}{2 \kappa_{1}}\right]+2 \rho \sigma_{1} \sigma_{2}\left(\frac{1-e^{-\kappa_{1} \tau}}{\kappa_{1}}\right)\left(\frac{1-e^{-\kappa_{2} \tau}}{\kappa_{2}}\right) \\
& {\left[\frac{1-e^{-\left(\kappa_{1}+\kappa_{2}\right)(T-t)}}{\kappa_{1}+\kappa_{2}}\right]+\sigma_{2}^{2}\left(\frac{1-e^{-\kappa_{2} \tau}}{\kappa_{2}}\right)^{2}\left[\frac{1-e^{-2 \kappa_{2}(T-t)}}{2 \kappa_{2}}\right] . }
\end{aligned}
$$

\section{Edgeworth expansion}

From Eq. (2.6), the calculation of the GAO value amounts to the determination of $P_{Q_{F_{n}}}\left[a_{R}(T)>g\right], n=0,1,2, \cdots\left(\right.$ note that $Q_{F_{n}}$ becomes $Q_{S}$ when $\left.n=0\right)$. Let $\pi^{(n)}(a)$ denote the density function of $a_{R}(T)$ under the measure $Q_{F_{n}}$. We estimate $P_{Q_{F_{n}}}\left[a_{R}(T)>g\right]$ by performing a cumulant expansion of $\pi^{(n)}(a)$. The cumulants of a distribution are related to the moments of a distribution. The first two cumulants of a distribution are simply the mean and variance of the distribution, and there exists an one-to-one relationship between moments and cumulants. Let $m_{j}^{(n)}$ and $c_{j}^{(n)}$ denote the $j^{\text {th }}$ moment and $j^{\text {th }}$ cumulants of $\pi^{(n)}(a)$. It is well known that (Collin-Dufresne and Goldstein, 2002)

$$
c_{1}^{(n)}=m_{1}^{(n)}, \quad c_{2}^{(n)}=m_{2}^{(n)}-\left(m_{1}^{(n)}\right)^{2}, \quad c_{3}^{(n)}=m_{3}^{(n)}-3 m_{1}^{(n)} m_{2}^{(n)}+2\left(m_{1}^{(n)}\right)^{3}, \text { etc. }
$$

We would like to approximate $P_{Q_{F_{n}}}\left[a_{R}(T)>g\right]$ in terms of the first three cumulants. Also, we illustrate how to find the first three moments by solving a system of Ricatti equations. By virtue of Eq. (2.6), we then obtain an approximate price formula of the GAO value based on the Edgeworth expansion of $\pi^{(n)}(a)$.

1. Approximation of $P_{Q_{F_{n}}}\left[a_{R}(T)>g\right]$ in terms of cumulants

Let $\Pi^{(n)}(\lambda)$ denote the characteristic function of $a_{R}(T)$ under $Q_{F_{n}}$, where

$$
\Pi^{n}(\lambda)=E_{Q_{F_{n}}}\left[e^{i \lambda a_{R}(T)}\right]=\int_{-\infty}^{\infty} e^{i \lambda a} \pi^{(n)}(a) d a
$$


The cumulants are defined to be the coefficients of a Taylor series expansion of the logarithm of the characteristic function, where

$$
\ln \Pi^{(n)}(\lambda)=\sum_{j=1}^{\infty} c_{j} \frac{(i \lambda)^{j}}{j !}
$$

By taking the Fourier inversion of $\Pi^{(n)}(\lambda)$ and keeping cumulants only up to the third order, we obtain

$$
\begin{aligned}
\pi^{(n)}(a) & =\frac{1}{2 \pi} \int_{-\infty}^{\infty} e^{-i \lambda a} \Pi^{(n)}(\lambda) d \lambda \\
& =\frac{1}{2 \pi} \int_{-\infty}^{\infty} \exp \left(-i \lambda a+i \lambda c_{1}^{(n)}-\frac{c_{2}^{(n)}}{2} \lambda^{2}-i \frac{c_{3}^{(n)}}{6} \lambda^{3}+o\left(\lambda^{3}\right)\right) d \lambda \\
& \approx \frac{1}{2 \pi} \int_{-\infty}^{\infty} \exp \left(-i\left(a-c_{1}^{(n)}\right) \lambda-\frac{c_{2}^{(n)}}{2} \lambda^{2}\right)\left(1-\frac{i c_{3}^{(n)}}{6} \lambda^{3}\right) d \lambda
\end{aligned}
$$

After some tedious integration procedure, we obtain

$$
\pi^{(n)}(a) \approx\left[\frac{1}{\sqrt{c_{2}^{(n)}}}-\frac{c_{3}^{(n)}\left(a-c_{1}^{(n)}\right)}{2\left(c_{2}^{(n)}\right)^{5 / 2}}+\frac{c_{3}^{(n)}\left(a-c_{1}^{(n)}\right)^{3}}{6\left(c_{2}^{(n)}\right)^{7 / 2}}\right] n\left(\frac{a-c_{1}^{(n)}}{\sqrt{c_{2}^{(n)}}}\right)
$$

where $n(x)=\frac{1}{\sqrt{2 \pi}} e^{-x^{2} / 2}$. Furthermore, we deduce that

$$
\begin{aligned}
P_{Q_{F_{n}}}[a(T)>g] & =\int_{g}^{\infty} \pi^{(n)}(a) d a \\
& \approx N\left(z_{1}\right)+\frac{c_{3}^{(n)}}{6\left(c_{2}^{(n)}\right)^{3 / 2}}\left(z_{1}^{2}-1\right) n\left(z_{1}\right),
\end{aligned}
$$

where

$$
z_{1}=\frac{c_{1}^{(n)}-g}{\sqrt{c_{2}^{(n)}}}
$$

2. Determination of the moments of $a_{R}(T)$ under the measure $Q_{F_{n}}$

We would like to find the $j^{\text {th }}$ moment of $a_{R}(T)$ under the measure $Q_{F_{n}}$ as defined by

$$
m_{j}^{(n)}=E_{Q_{F_{n}}}\left[a(T)^{j}\right]
$$


Note that

$$
\begin{aligned}
a(T)^{j} & =\left[\sum_{n=0}^{\omega-R-1}{ }_{n} p_{R} D_{T+n}(T)\right]^{j} \\
& =\sum_{n_{1}, n_{2}, \cdots, n_{j}=0}^{\omega-R}\left(n_{1} p_{R} n_{2} p_{R} \cdots{ }_{n_{j}} p_{R}\right)\left[D_{T+n_{1}}(T) D_{T+n_{2}}(T) \cdots D_{T+n_{j}}(T)\right]
\end{aligned}
$$

so that

$$
\begin{aligned}
m_{j}^{(n)}= & \sum_{n_{1}, n_{2}, \cdots, n_{j}=0}^{\omega-R-1}\left({ }_{n} p_{R} n_{2} p_{R} \cdots n_{j} p_{R}\right) \\
& E_{Q_{F_{n}}}\left[\exp \left(-\sum_{k=1}^{j}\left[\boldsymbol{A}_{T+n_{k}}(T)^{T} \boldsymbol{x}(T)+B_{T+n_{k}}(T)\right]\right)\right] .
\end{aligned}
$$

The moments are seen to have the exponential affine form. For nice analytical tractability associated with the Gaussian type models, we assume that the drift term $\boldsymbol{\mu}(\boldsymbol{x}, t)$ takes the linear form

$$
\boldsymbol{\mu}(\boldsymbol{x}, t)=\boldsymbol{\mu}_{0}(t)+\mu_{1}(t) \boldsymbol{x},
$$

where $\boldsymbol{\mu}_{0}(t)$ is a $\ell$-component vector and $\mu_{1}(t)$ is a $\ell \times \ell$ matrix. The expectation term in Eq. (4.7) can be evaluated by solving a system of Ricatti equations. obtain

By following the standard evaluation procedures in affine term structure models, we

$$
\begin{aligned}
& E_{Q_{F_{n}}}\left[\exp \left(-\sum_{k=1}^{j} \boldsymbol{A}_{T+n_{k}}(T)^{T} \boldsymbol{x}(T)-B_{T+n_{k}}(T)\right)\right] \\
= & \exp \left(-\boldsymbol{G}_{T}(t)^{T} \boldsymbol{x}(t)-G_{T}^{0}(t)\right)
\end{aligned}
$$

where $\boldsymbol{G}_{T}(t)$ and $G_{T}^{0}(t)$ have dependence on $n_{1}, n_{2}, \cdots, n_{j}$ and $n$, and they satisfy the following systems of Ricatti equations.

(i)

$$
\begin{aligned}
& \frac{d \boldsymbol{G}_{T}(t)}{d t}+\mu_{1}(t)^{T} \boldsymbol{G}_{T}(t)=\mathbf{0} \\
& \boldsymbol{G}_{T}(T)=\sum_{k=1}^{j} \boldsymbol{A}_{T+n_{k}}(T)
\end{aligned}
$$

(ii) 


$$
\begin{aligned}
& \frac{d G_{T}^{0}}{d t}+\boldsymbol{G}_{T}(t)^{T}\left\{\boldsymbol{\mu}_{0}(t)+\sigma(t) \sigma_{S}(t)+\sigma(t) \sigma(t)^{T}\left[\boldsymbol{A}_{T}(t)-\boldsymbol{A}_{T+n}(t)\right]\right\} \\
= & \frac{1}{2} \boldsymbol{G}_{T}(t)^{T} \sigma(t) \sigma(t)^{T} \boldsymbol{G}_{T}(t) \\
& G_{T}^{0}(T)=\sum_{k=1}^{j} B_{T+n_{k}}(T) .
\end{aligned}
$$

Let $\Phi_{T}(t)$ be the solution to the following system of differential equations

$$
\begin{aligned}
& \frac{d \Phi_{T}(t)}{d t}=-\mu_{1}(t)^{T} \Phi_{T}(t) \\
& \Phi_{T}(T)=I
\end{aligned}
$$

where $\Phi_{T}(t)$ is a $\ell \times \ell$ matrix and $I$ is the $\ell \times \ell$ identity matrix. It can be shown that

$$
\Phi_{T}(t)=\exp \left(\int_{t}^{T} \mu_{1}(u)^{T} d u\right) .
$$

Now, the closed form solution to $\boldsymbol{G}_{T}(t)$ and $G_{T}^{0}(t)$ can be expressed in terms of $\Phi_{T}(t)$ as follows

$$
\begin{aligned}
\boldsymbol{G}_{T}(t)= & \Phi_{T}(t) \boldsymbol{G}_{T}(T)=\sum_{k=1}^{j} \exp \left(\int_{t}^{T} \mu_{1}(u)^{T} d u\right) \boldsymbol{A}_{T+n_{k}}(T) \\
G_{T}^{0}(t)= & G_{T}^{0}(T)+\int_{t}^{T} \boldsymbol{G}_{T}(u)^{T}\left\{\boldsymbol{\mu}_{0}(u)+\sigma(u) \boldsymbol{\sigma}_{S}(u)\right. \\
& \left.+\sigma(u) \sigma(u)^{T}\left[\boldsymbol{A}_{T}(u)-\boldsymbol{A}_{T+n}(u)-\frac{\boldsymbol{G}_{T}(u)}{2}\right]\right\} d u .
\end{aligned}
$$

\subsection{Two-factor Gaussian model}

We illustrate how to compute $\boldsymbol{G}_{T}(t)$ and $G_{T}^{0}(t)$ using the two-factor Gaussian interest rate model defined by Eqs. (3.13a,b) and the equity fund dynamics defined by Eqs. (3.15a,b). The volatility matrix $\sigma(t)$ is given by

$$
\sigma(t)=\left(\begin{array}{ccc}
\sigma_{1} & 0 & 0 \\
\sigma_{2} \rho & \sigma_{2} \sqrt{1-\rho^{2}} & 0
\end{array}\right)
$$

so that

$$
\sigma(t) \sigma(t)^{T}=\left(\begin{array}{cc}
\sigma_{1}^{2} & \rho \sigma_{1} \sigma_{2} \\
\rho \sigma_{1} \sigma_{2} & \sigma_{2}^{2}
\end{array}\right)
$$


The solution to $\Phi_{T}(t)$ is found to be

$$
\Phi_{T}(t)=\left(\begin{array}{cc}
e^{-\kappa_{1}(T-t)} & 0 \\
0 & e^{-\kappa_{2}(T-t)}
\end{array}\right)
$$

while $\boldsymbol{A}_{T}(t)$ and $B_{T}(t)$ are given by Eqs. $(3.14 \mathrm{a}, \mathrm{b})$.

Finally, the solution to $\boldsymbol{G}_{T}(t)$ and $G_{T}^{0}(t)$ are given by

$$
\boldsymbol{G}_{T}(t)=\left(\begin{array}{c}
G_{T, 1}(T) e^{-\kappa_{1}(T-t)} \\
G_{T, 2}(T) e^{-\kappa_{2}(T-t)}
\end{array}\right) \text { where } \boldsymbol{G}_{T}(T)=\left(\begin{array}{c}
G_{T, 1}(T) \\
G_{T, 2}(T)
\end{array}\right)
$$

and

$$
\begin{aligned}
G_{T}^{0}(t)= & G_{T}^{0}(T)+\sigma_{1} \sigma_{S} \rho_{S 1} G_{T, 1}(T)\left(\frac{1-e^{-\kappa_{1}(T-t)}}{\kappa_{1}}\right) \\
& +\sigma_{2} \sigma_{S}\left(\rho_{S 1} \rho+\rho_{S 2} \sqrt{1-\rho^{2}}\right) G_{T, 2}(T)\left(\frac{1-e^{-\kappa_{2}(T-t)}}{\kappa_{2}}\right) \\
& -\sigma_{1}^{2} G_{T, 1}(T)\left[\frac{1-e^{-\kappa_{1} n}}{\kappa_{1}}+\frac{G_{T, 1}(T)}{2}\right]\left(\frac{1-e^{-2 \kappa_{1}(T-t)}}{2 \kappa_{1}}\right) \\
& -\sigma_{2}^{2} G_{T, 2}(T)\left[\frac{1-e^{-\kappa_{2} n}}{\kappa_{2}}+\frac{G_{T, 2}(T)}{2}\right]\left(\frac{1-e^{-2 \kappa_{2}(T-t)}}{2 \kappa_{2}}\right) \\
& -\rho \sigma_{1} \sigma_{2}\left[G_{T, 1}(T)\left(\frac{1-e^{-\kappa_{2} n}}{\kappa_{2}}\right)+G_{T, 2}(T)\left(\frac{1-e^{-\kappa_{1} n}}{\kappa_{1}}\right)\right. \\
& \left.+G_{T, 1}(T) G_{T, 2}(T)\right]\left[\frac{1-e^{-\left(\kappa_{1}+\kappa_{2}\right)(T-t)}}{\kappa_{1}+\kappa_{2}}\right] .
\end{aligned}
$$

\section{Affine approximation approach}

Unlike the Edgeworth expansion approach, Singleton and Umantsev (2002) propose to approximate the probability of exercising the option $P_{Q_{F_{n}}}\left[a_{R}(x, T)>g\right]$ through an approximation of the exercise region itself. They show that if all the future cashflows are positive, then the boundary of the in-the-money region $\left\{a_{R}(\boldsymbol{x}, T)>g\right\}$ is a concave surface. Their method involves the linearization of the exercise boundary by fitting a hyperplane $\boldsymbol{\beta}^{T} \boldsymbol{x}=1$ that approximates the exercise boundary $a_{R}(\boldsymbol{x}, T)=g$. The probability of exercising $P_{Q_{F_{n}}}\left[a_{R}(\boldsymbol{x}, t)>g\right]$ is then approximated by either $P_{Q_{F_{n}}}\left[\boldsymbol{\beta}^{T} \boldsymbol{x}>1\right]$ or $P_{Q_{F_{n}}}\left[\boldsymbol{\beta}^{T} \boldsymbol{x}<1\right]$ (whose choice depends on the location of the exercise region). For the Gaussian type models, $\boldsymbol{\beta}^{T} \boldsymbol{x}(T)=\beta_{1} x_{1}(T)+\cdots+\beta_{\ell} x_{\ell}(T)$ is normally distributed whose mean and variance are given by $\boldsymbol{\beta}^{T} \overline{\boldsymbol{\mu}}_{\boldsymbol{x}}$ and $\boldsymbol{\beta}^{T} \sigma_{\boldsymbol{x}} \boldsymbol{\beta}$, where $\overline{\boldsymbol{\mu}}_{\boldsymbol{x}}$ and $\sigma_{\boldsymbol{x}}$ are the conditional mean vector and covariance matrix of $\boldsymbol{x}(T)$ given $\boldsymbol{x}(t)$ under $Q_{F_{n}}$.

\subsection{Fitting algorithm}


Consider a two-factor interest rate model with two risk factors, the fitting algorithm involves the following steps.

1. Choose a level of significance $\alpha$ (say, $1 \%$ ), then find the two values $x_{2, \alpha / 2}$ and $x_{2,1-\alpha / 2}$ such that

$$
P_{Q_{F_{n}}}\left[x_{2, \alpha / 2}<x_{2}(T)<x_{2,1-\alpha / 2}\right]=1-\alpha .
$$

2. Once $x_{2, \alpha / 2}$ and $x_{2,1-\alpha / 2}$ are known, solve for $x_{1, \alpha / 2}$ and $x_{1,1-\alpha / 2}$ so that the two points $\left(x_{1, \alpha / 2}, x_{2, \alpha / 2}\right)$ and $\left(x_{1,1-\alpha / 2}, x_{2,1-\alpha / 2}\right)$ fall on the exercise boundary: $a(x, T)=$ $g$.

3. Fit a hyperplane (degenerates to a line in the case of a two-factor interest rate model)

$$
\beta_{1} x_{1}+\beta_{2} x_{2}=1
$$

to the two points determined in Step 2 by solving for the parameters $\beta_{1}$ and $\beta_{2}$ through

$$
\boldsymbol{\beta}=\left(\begin{array}{c}
\beta_{1} \\
\beta_{2}
\end{array}\right)=\left(\begin{array}{cc}
x_{1, \alpha / 2} & x_{2, \alpha / 2} \\
x_{1,1-\alpha / 2} & x_{2,1-\alpha / 2}
\end{array}\right)^{-1}\left(\begin{array}{l}
1 \\
1
\end{array}\right) \text {. }
$$

Choose the appropriate region $\left\{\boldsymbol{\beta}^{T} \boldsymbol{x}>1\right\}$ or $\left\{\boldsymbol{\beta}^{T} \boldsymbol{x}<1\right\}$ so as to approximate the exercise region $\left\{a_{R}(\boldsymbol{x}, t)>g\right\}$.

\section{Numerical results}

In this section, we present our numerical experiments that were performed to compare numerical accuracy and computational efficiency of the three analytic approximation methods. Also, we explore how the GAO value depends on the guaranteed conversion rate $g$ and various correlation coefficients in the pricing model.

In our numerical calculations, we use the following set of parameter values in the pricing model (unless otherwise specified).

Parameters in the equity and interest rate models

$$
\begin{aligned}
& S_{t}=100, \quad q=5 \%, \quad \sigma_{S}=10 \%, \quad \kappa_{1}=0.77, \quad \kappa_{2}=0.08, \\
& \sigma_{1}=2 \%, \quad \sigma_{2}=1 \%, \quad \rho=-0.7, \quad \rho_{S 1}=0.5, \quad \rho_{S 2}=0.5 \text {. }
\end{aligned}
$$

Current yield curve, $Y(T)=r_{0}+0.04\left(1-e^{-0.2 T}\right)$ where $r_{0}$ is taken to assume different constant values. 
Mortality data

retirement age $=65$, maximum age $=100$

\begin{tabular}{c||l}
$n$ & ${ }_{n} P_{R}$ \\
\hline 1 & 0.9871 \\
\hline 2 & 0.9730 \\
\hline 3 & 0.9578 \\
\hline 4 & 0.9411 \\
\hline 5 & 0.9229 \\
\hline 6 & 0.9029 \\
\hline 7 & 0.8808 \\
\hline 8 & 0.8567 \\
\hline 9 & 0.8304 \\
\hline 10 & 0.8018 \\
\hline 11 & 0.7708 \\
\hline 12 & 0.7374 \\
\hline 13 & 0.7015 \\
\hline 14 & 0.6632 \\
\hline 15 & 0.6226 \\
\hline 16 & 0.5798 \\
\hline 17 & 0.5351 \\
\hline 18 & 0.4889
\end{tabular}

\begin{tabular}{c||l}
$n$ & ${ }_{n} P_{R}$ \\
\hline 19 & 0.4414 \\
\hline 20 & 0.3934 \\
\hline 21 & 0.3454 \\
\hline 22 & 0.2981 \\
\hline 23 & 0.2523 \\
\hline 24 & 0.2088 \\
\hline 25 & 0.1684 \\
\hline 26 & 0.1319 \\
\hline 27 & 0.0998 \\
\hline 28 & 0.0725 \\
\hline 29 & 0.0503 \\
\hline 30 & 0.0330 \\
\hline 31 & 0.0203 \\
\hline 32 & 0.0115 \\
\hline 33 & 0.0059 \\
\hline 34 & 0.0027 \\
\hline 35 & 0.0011
\end{tabular}

Other parameters

$g=9$, time to expiry $=T-t=15$ (years),

significant level in the affine approximation $=0.01$,

100, 000 trial runs were performed in each Monte Carlo simulation,

Edgeworth expansion was taken up to the third order.

\subsection{Computational efficiency and numerical accuracy}

In a typical run in the numerical calculations of the GAO value at a given chosen value of $r_{0}$, the following computer running times (in minutes) for various methods are recorded in Table 1. Since tedious iterative calculations are required to calculate the higher order moments in the Edgeworth expansion method, our experience showed that even only up to the third order expansion, the running time required by the Edgeworth expansion is longer than that of the Monte Carlo simulation with 100,000 trials. Since closed form formulas are available in the minimum variance duration method, the required running time was significantly shorter than that of the other numerical methods. 


\begin{tabular}{l|c|c|c|c} 
method & Monte Carlo simulation & Edgeworth expansion & affine approximaton & min. var. duration \\
\hline running time & 0.4305 & 1.136 & 0.1812
\end{tabular}

Table 1 Comparison of computer running time (in minutes) of various numerical methods that compute the GAO value.

In Table 2, we list the numerical results of GAO value obtained by various numerical methods at different choices of $r_{0}$. We observe acceptable agreement between the numerical values.

\begin{tabular}{|c|c|c|c|c|}
\hline$r_{0} \%$ & Monte Carlo Simulation & Edgeworth expansion & affine approximation & min. var. duration \\
\hline 0.5 & 11.7750 & 11.8161 & 11.7913 & 11.8100 \\
\hline 1.0 & 9.7568 & 9.7502 & 9.7412 & 9.7714 \\
\hline 1.5 & 7.8952 & 7.8479 & 7.8529 & 7.8958 \\
\hline 2.0 & 6.1543 & 6.1293 & 6.1418 & 6.1946 \\
\hline 2.5 & 4.6735 & 4.6199 & 4.6313 & 4.6860 \\
\hline 3.0 & 3.3793 & 3.3408 & 3.3464 & 3.3911 \\
\hline 3.5 & 2.3257 & 2.2999 & 2.3044 & 2.3273 \\
\hline 4.0 & 1.5116 & 1.4897 & 1.5057 & 1.5008 \\
\hline 4.5 & 0.9222 & 0.8942 & 0.9310 & 0.9008 \\
\hline 5.0 & 0.5201 & 0.4922 & 0.5439 & 0.4984 \\
\hline
\end{tabular}

Table 2 Comparison of numerical results of GAO value obtained by various numerical methods.

We also performed numerical experiments to explore the pricing errors of the three analytic approximation methods. Using the Monte Carlo results as the benchmark, we calculated the GAO value at 100 different value of $r_{0}\left(r_{0}=0.1 \%, 0.2 \%, \cdots, 10 \%\right)$ and computed the percentage error of each analytic approximation method. The variation of the percentage error with respect to $\frac{\bar{a}}{g D_{T}(t)}$ is plotted in Fig. 1. The pricing error is typically less than $1 \%$ when $\frac{\bar{a}}{g D_{T}(t)}>1$ (the annuity option is currently in-the-money) while the accuracy deteriorates when $\frac{\bar{a}}{g D_{T}(t)}$ falls below 1. Similar behaviors on numerical accuracy are exhibited in swaption calculations using the affine approximation method (Singleton and Umantsev, 2002) and minimum variance duration method (Munk, 1999).

\subsection{Pricing behaviors of the guaranteed annuity option}


We investigate the pricing behaviors of the GAO with respect to various parameters in the pricing model. In Fig. 2, we plot the GAO value against $g$ at varying values of $T-t$. The curves exhibit consistency with the intuition that the GAO value should be a decreasing function of $g$. The rate of decrease of GAO value is higher at a lower value of $g$. Also, the GAO has a higher value when the policyholder enters the contract closer to retirement (smaller value of $T-t$ ). This is related to the time value of money since smaller $T-t$ means shorter time horizon over which the annuity payments are discounted. This effect counteracts the usual theta effect of option value, where a longer-lived option usually has a higher value.

Our GAO pricing model assumes that the interest rate dynamics is governed by two risk factors $(\mathrm{G} 2++$ model). Therefore, there are 3 correlation coefficients in the model, namely, the correlation coefficient $\rho$ between the interest rate risk factors, the correlation coefficients $\rho_{S 1}$ and $\rho_{S 2}$ between the stock price process and the risk factors. In Fig. 3, we plot the GAO value against $\rho$ with different sets of values of $\rho_{S 1}$ and $\rho_{S 2}$. In the analytic approximation price formula, the functional dependence of the GAO value on these correlation coefficients appears to be quite complicated. From the plots in Fig. 3, it is quite disquieting to observe that the GAO value is highly sensitive to the correlation coefficients. Similar phenomena of price sensitivity to the correlation coefficient have also been reported by Ballotta and Haberman (2003a).

\section{Conclusions}

Since there is no closed form analytic price formula for a guaranteed annuity option when the interest rate dynamics is modeled by a multi-factor affine term structure model, the numerical valuation of the guarantee in deferred annuity pension policies is resorted to either Monte Carlo simulation or analytic approximation methods. In this paper, we construct three analytic approximation methods for efficient valuation of the annuity option value when the interest rate dynamics is modeled by a two-factor Gaussian interest rate model. The method of minimum variance duration starts with a judicious analytic approximation so that closed form formula can be obtained. The pricing error is minimized by choosing the period $\tau$ of a reference bond such that the variance of the value of the annuity payment normalized by the price of the $(T+\tau)$-maturity bond is minimized. The Edgeworth expansion method seeks the Edgeworth approximation of the probability distribution of the annuity value at option's maturity. In the affine approximation, the exercise probability of the annuity option is approximated through the approximation of the concave exercise boundary by a hyperplane. Comparing these three analytic approximation methods in terms of numerical accuracy and computational efficiency, the method of minimum variance duration seems to have the best performance among them. When the annuity option is in-the-money or slightly out-of-the-money, the pricing error of these approximation methods are within a few percentage points. Though the three-term Edgeworth expansion demonstrates sufficient accuracy, the computational time required is even longer than that of the Monte Carlo simulation method using 100, 000 simulation paths. 
Our numerical studies showed that the value of the annuity option is highly dependent on the guaranteed conversion rate of the annuity and the correlation coefficients among the risk factors.

\section{References}

Ballotta, L., Haberman, S., 2003a. Valuation of guaranteed annuity conversion options. Insurance: Mathematics and Economics 33, 87-108.

Ballotta, L., Haberman, S., 2003b. The fair valuation problem of guaranteed annuity options: the stochastic mortality environment case. Working paper of City University, London.

Biffis, E., Millossovich, P., 2006. The fair value of guaranteed annuity options. Scandinavian Actuarial Journal, First issue, 23-41.

Boyle, P., Hardy, M., 2003. Guaranteed annuity options. ASTIN Bulletin 33(2), 125-152.

Collin-Dufresne, P., Goldstein, R.S., 2002. Pricing swaptions within an affine framework. Journal of Derivatives, Fall issue, 1-18.

Cox, J.C., Ingersoll, J.E., Ross, S.A., 1979. Duration and the measurement of basis risk. Journal of Business 51(1), 51-61.

Dai, Q., Singleton, K.J., 2000. Specification analysis of affine term structure models. Journal of Finance 55, 1943-1978.

Heath, D., Jarrow, R., Morton, A., 1992. Bond pricing and term structure of interest rates: a new methodology for contingent claims valuation. Econometrica 60(1), 77-105.

Jamshidian, F., 1989. An exact bond option formula. Journal of Finance, 44(1), 205-209.

Munk, C., 1999. Stochastic duration and fast coupon bond option pricing in multi-factor models. Reviews of Derivatives Research 3, 157-181.

O'Brien, C., 2001. Guaranteed annuity options: Five issues for resolution. Working paper of University of Nottingham.

Pelsser, A., 2003. Pricing and hedging guaranteed annuity options via static option replication. Insurance: Mathematics and Economics 33(2), 283-296. 
Singleton, K.J., Umantsev, L., 2002. Pricing coupon-bond options and swaptions in affine term structure models. Mathematical Finance 12(4), 427-446.

Wei, J., 1997. A simple approach to bond option pricing. Journal of Futures Markets 17(2), 131-160.

Wilkie, A.D., Waters, H.R., Kong, S., 2004. Reserving, pricing and hedging for policies with guaranteed annuity options. British Actuarial Journal 9(2), 263-425. 


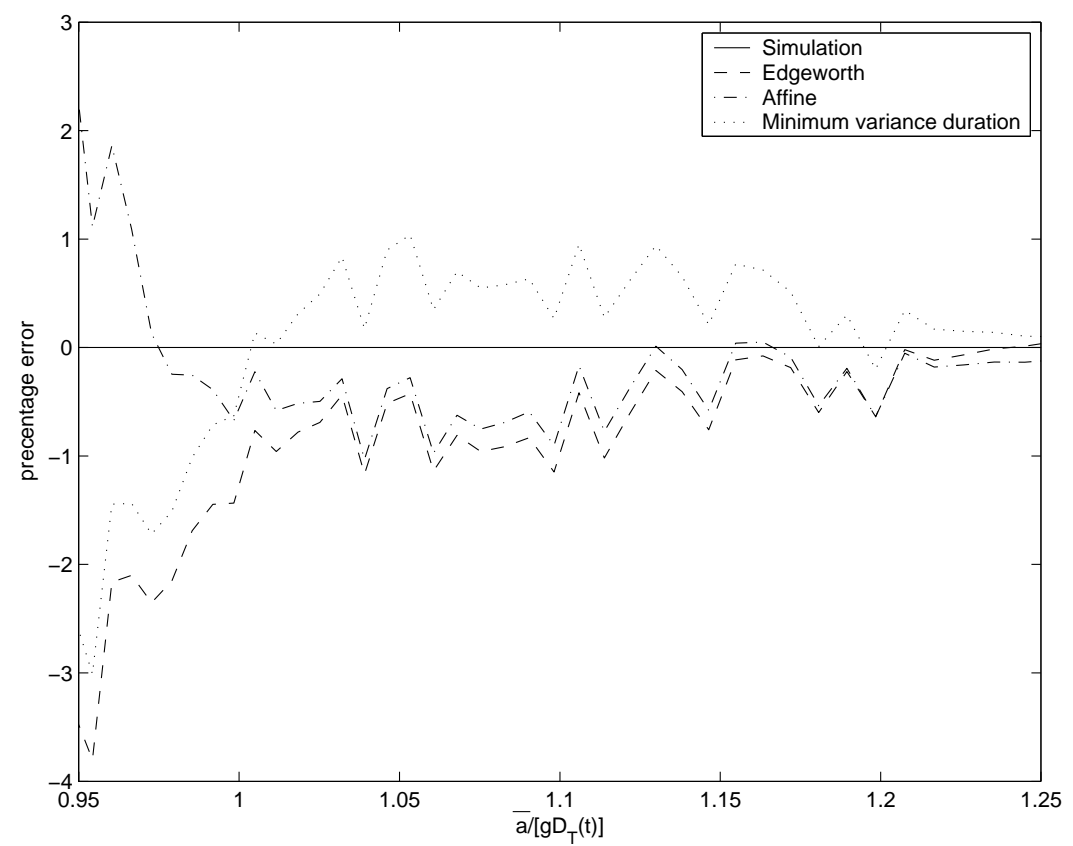

Fig. 1. Comparison of the pricing error (in percentage) of various analytic approximation methods. Good numerical accuracy is observed when $\frac{\bar{a}}{g D_{T}(t)}>1$ (the annuity option is currently in-the-money).

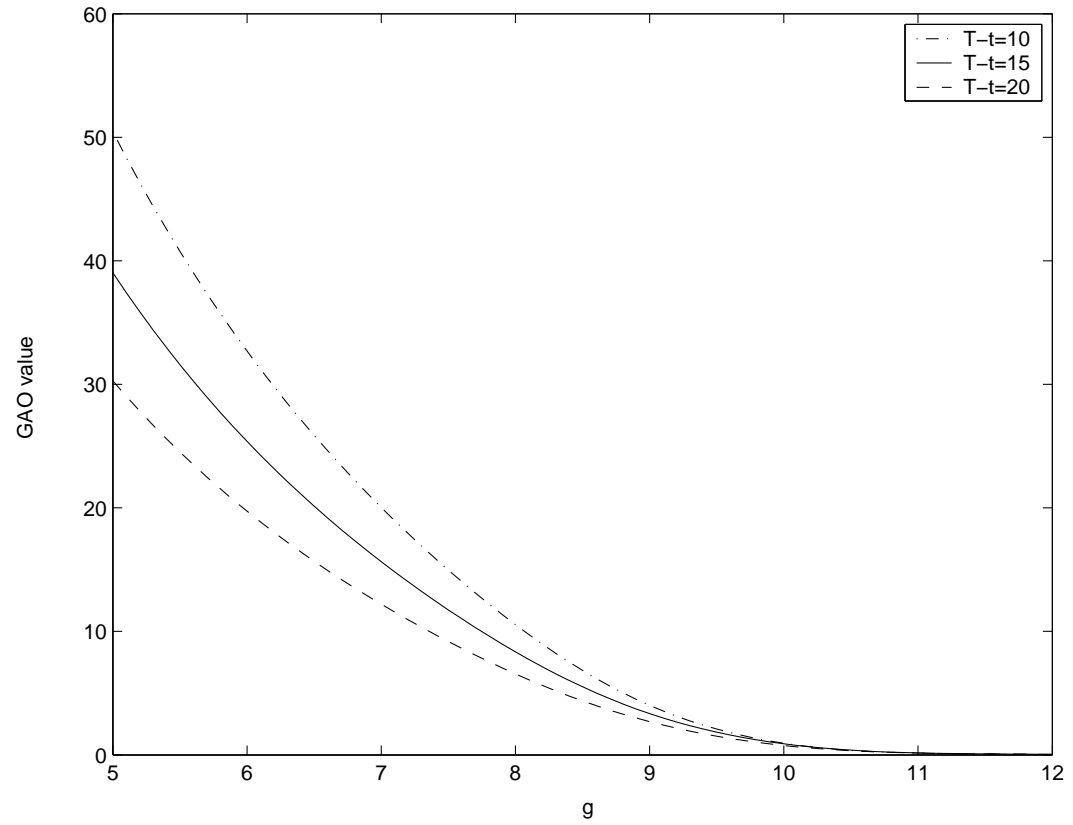

Fig. 2. Plot of GAO value against guaranteed conversion rate $g$ at varying values of time to expiry $T-t$. 


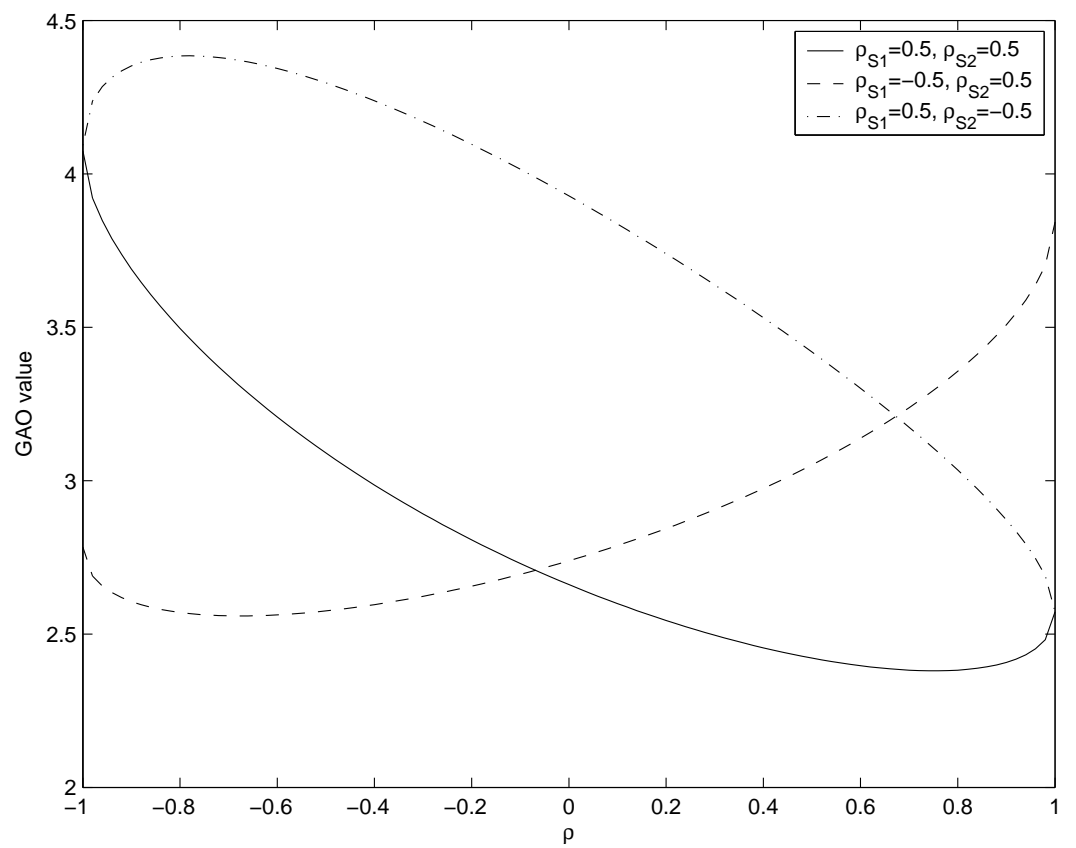

Fig. 3. Price sensitivity of the GAO value to the correlation coefficients in the pricing model. 